\title{
14
}

\section{A comparative study of hydrogen equations of state}

\author{
D. SAUMON
}

Lunar and Planetary Laboratory, L'niversity of Arizona, Tucson, AZ 85721, LSA

\begin{abstract}
The numerous complexities underlying large tables of thermodynamic quantities act as a deterrent to a careful evaluation of their reliability. As a consequence, equations of state are often used as black boxes. To clarify this situation, some of the more critical issues of equation of state physics are discussed from the point of view of the user. They are illustrated by a comparison of four equations of state for hydrogen. The flaws and disagreements thus brought into light are explained and evaluated with simple physical arguments.
\end{abstract}

Les tables d'équations d'état utilisées en astrophysique découlent de modèles d'une complexité telle qu'il est souvent difficile d'en évaluer la fiabilité. Il en résulte une situation où les équations d'état sont souvent utilisées sans une analyse critique de leur contenu physique ni de leur précision. Dans le but de remédier à cette situation, une discussion des principaux éléments physiques des équations d'état est présentée dans l'optique de l'utilisateur. Quatre équations d'état de l'hydrogène développées pour être appliquées à des problèmes d'astrophysique stellaire sont comparées de façon critique. Cette comparaison illustre l'importance de certains éléments clés des équations d'état et la nature des problèmes qui subsistent. Les défauts et les différences observés entre ces quatre équations d'état sont élucidés en termes de physique de base. 


\subsection{Introduction}

The richness of stellar phenomena exposed by modern observational techniques calls for a quantitative understanding of more subtle, "second order" effects in stellar structure. Examples of phenomena requiring accurate modeling of the underlying physics include the solar oscillation spectrum, the solar neutrino problem, stellar pulsations, and the origin of the abundance of elements in photospheres of white dwarfs.

The equation of state (EOS) $\dagger$ constitutes one of the properties of matter, along with transport coefficients, which enter the equations of stellar structure (Clayton 1983, p. 436ff). The pressure $P(\rho, T)$ and the entropy $S(\rho, T)$ appear explicitly in these equations and dictate the mechanical and thermal equilibria of the star, respectively. Beyond the fact that an equation of state is necessary to compute a stellar model, the quantitative understanding of "subtle" stellar phenomena does depend, sometimes sensitively, on the assumed EOS (see for example, the review by Däppen 1994). The importance of this point is not always realized.

The astrophysicist interested in EOS as an input to solve a particular problem is faced with a number of difficult choices. Most applications require such complex EOS that it is not practical to compute one locally. This raises a number of questions which are often overlooked. Which of the available EOS is most appropriate for a particular problem? Does it include the proper physics? Which one is the most reliable? How does it compare with other EOS? Because EOS calculations usually involve a large number of approximations and assumptions as well as some level of internal inconsistency, it is very difficult to answer these questions from published literature alone. In this context, a direct comparison of several EOS tables and of the underlying assumptions becomes a powerful tool to reveal flaws and poor approximations and to develop a healthy appreciation of the uncertainties which persist in some physical regimes.

The complexity of an EOS calculation increases considerably when nonideal effects are introduced. In fact, such calculations can only be performed numerically, and the results usually presented in tabular form. Historically, the Lawrence Livermore National Laboratory and the Los Alamos Scientific Laboratory have invested much effort in the development of tabular equations of state which are frequently used in astrophysical applications. Significant progress in the computation of realistic equations of state under

† Strictly speaking, the equation of state is the relation between pressure, te mperature and density, $P(\rho, T)$. In the context of this work, we loosely apply the term to the ensemble of equilibrium thermodynamic properties of matter, such as the entropy, internal energy, specific heat, etc. 
stellar conditions was made by Graboske, Harwood and Rogers (1969), Kerley (1972), Fontaine, Graboske and Van Horn (1977), Lamb (1974), Lamb and Van Horn (1975), Magni and Mazzitelli (1979) and more recently, by Rogers (1981), Hummer, Mihalas and Däppen (Hummer and Mihalas 1988; Mihalas, Däppen and Hummer 1988; Däppen et al. 1988) and under conditions typical of the interior of giant planets by Stevenson (1975), Stevenson and Salpeter (1977), Hubbard and DeWitt (1985, and references therein), and Marley and Hubbard (1988).

The past decade has seen tremendous progress in our understanding of dense matter physics, on both the experimental and theoretical fronts. Equations of state for dense plasmas are now becoming well understood, thanks in part to progress in computer technology, which has permitted simulations of ever increasing complexity. These studies have demonstrated the great utility of a variety of approximations for the computation of plasma properties. In particular, when a sample of any substance is sufficiently compressed, atoms (or molecules) are so closely packed that the exclusion principle promotes bound electrons into conducting states (for hydrogen, this occurs near $1 \mathrm{~g} / \mathrm{cm}^{3}$ ). This "pressure ionization" represents a thorny problem in the calculation of an equation of state. It is often avoided by simply interpolating between atomic and fully ionized limits. Recent advances in statistical physics offer the opportunity for significant improvements in our understanding of this poorly understood phenomenon.

Although great progress is being made in the laboratory, most astrophysically interesting regimes are still weakly constrained by experimental data. Thus the validity of an EOS can only be established in an indirect fashion. It should, of course, reproduce known asymptotic limits. Computer simulations also provide useful but limited tests for theoretical equations of state. In addition, the EOS is subject to the fundamental thermodynamic constraints of mechanical and thermal stability,

$$
\left.\frac{\partial P}{\partial V}\right|_{T}<0, \quad \text { and }\left.\quad \frac{\partial S}{\partial T}\right|_{V}>0
$$

respectively, and of thermodynamic consistency,

$$
\left.\frac{\partial P}{\partial T}\right|_{V}=\left.\frac{\partial S}{\partial V}\right|_{T}
$$

where $P$ and $S$ represent the total pressure and entropy of a system occupying a volume $V$ at temperature $T$.

Equations of state which satisfies these constraints is not necessarily accurate. In regimes dominated by non-ideal effects, and where neither experiments nor computer simulations are available, it is very difficult to establish 
their validity. Nevertheless, the magnitude of uncertainties and their relative merit can be estimated by comparing independent EOS calculations and understanding the effects of the respective underlying assumptions.

In this review, we have chosen four hydrogen EOS for a detailed comparison of their respective thermodynamic variables. Hydrogen has a rich phase diagram and illustrates most of the situations encountered under astrophysical conditions. It is the most abundant element in the universe and constitutes $75 \%$ of the mass of the majority of stars. It is therefore representative of the bulk of stellar matter. The choice of a pure composition, as opposed to a cosmic mixture of hydrogen and helium, for example, greatly simplifies the discussion of the underlying physics. One of these EOS (Saumon and Chabrier 1991, 1992) is not fully published and a few remarks relevant to this comparison are given below. But first, we briefly review the phase diagram of hydrogen in an astrophysical context as well as the most frequently used method for computing EOS, the free energy minimization technique, on which all four EOS discussed here are based.

\subsection{The phase diagram of hydrogen}

Phase diagrams play an essential role in illuminating the EOS physics relevant to a particular problem. The simplified phase diagram for hydrogen of Fig. 1 helps to make a few basic points. In the low-density, lowtemperature region, hydrogen is essentially neutral and forms atoms and molecules. Molecules dominate at low temperatures $(\log T<3.5) \dagger$ and they dissociate into atoms as the temperature is raised. At still higher temperatures, atoms ionize to form a low-density plasma of protons and electrons. The dashed curve delimiting these three regions indicate a degree of dissociation (or ionization) of $50 \%$ and is based on detailed chemical equilibrium calculations for a non-ideal mixture of $\mathrm{H}_{2}, \mathrm{H}, \mathrm{H}^{+}$and $e$. At densities above $\log \rho \approx-2$, atoms and molecules interact strongly and form a non-ideal fluid. In addition, the Saha equations become inappropriate for $\log \rho>-1$, so that it is not possible to estimate the chemical equilibrium in this dense fluid with simple theories. At even higher densities, near $\log \rho=0$ for hydrogen, the mean distance between $\mathrm{H}$ atoms becomes comparable to twice the value of the Bohr radius and the electronic wave functions of neighboring atoms overlap. The electrons are forced into unbound states and the fluid becomes a pressure ionized plasma. A calculation of pressure ionization by Saumon and Chabrier (1992) reveals that pressure ionization

† Throughout this work, $\log T$ is the logarithm of the temperature in $K$, and $\log \rho$ is the logarithm of the mass density in $\mathrm{g} / \mathrm{cm}^{3}$. 


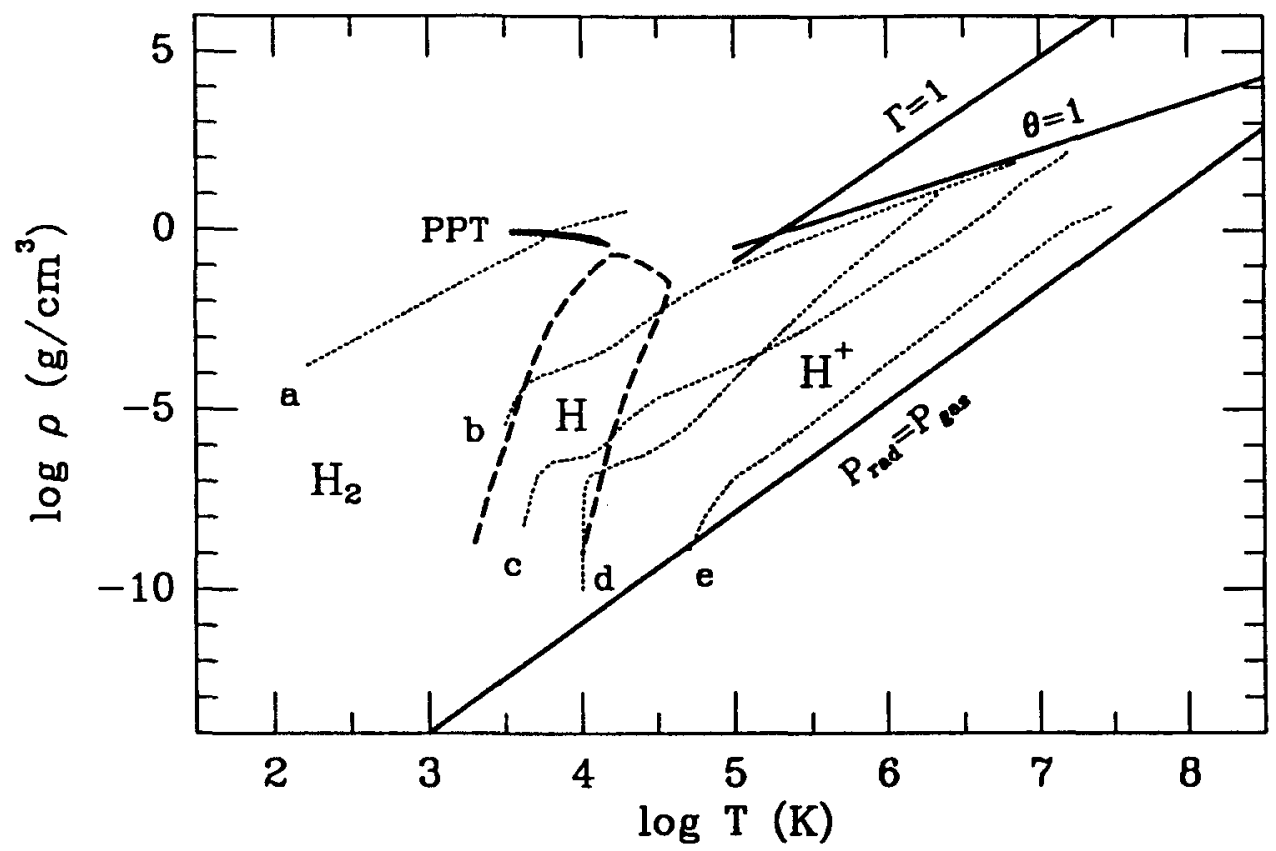

Fig. 14.1 Phase diagram for hydrogen. Heavy solid lines separate various physical regimes. Below the $P_{\text {rad }}=P_{\text {gaa }}$ line, the pressure of the photon gas exceeds that of the matter $\left(\mathrm{H}^{+}\right.$and $e$ ). Electrons are degenerate above the $\theta=1$ line, and protons form a strongly coupled plasma above the $\Gamma=1$ line. The thick curve labeled PPT shows the metastable region of the Plasma Phase Transition. The abundance of atomic hydrogen, $H$, is $50 \%$ along the dashed curve which indicates regimes of partial dissociation and ionization. The dotted curves are interior models for a) Jupiter, b) $0.3 M_{\odot}$ main sequence star, c) the Sun, d) the outer hydrogen layer of a $T_{\text {eff }}=12500 \mathrm{~K}$ DA white dwarf and e) a $15 M_{\odot}$ main sequence star.

of hydrogen may not be a gradual process at all temperatures but could occur discontinuously through a first order phase transition, the so-called plasma phase transition (PPT). The metastable region of this transition is shown by the curved labeled "PPT" and ends at a critical temperature of $\log T_{c}=4.185$.

Two important issues pertaining to the plasma are the degree of electron degeneracy and the strength of the Coulomb coupling between the charged particles. Above the solid line labeled $\theta=1$, where $\theta=\epsilon_{F} / k T$, the Fermi energy of the electrons $\epsilon_{F}$ is larger than $k T$, and they are therefore degenerate. Protons, on the other hand, remain classical over most of this diagram and in all astrophysical conditions (except in neutron stars). Above the line $\Gamma=1$, non-ideal Coulomb effects play an important role as the electrostatic potential energy between two protons, $e^{2} / a$, where $e$ is the 
charge quantum and $a$ is the mean interparticle distance, becomes larger than their kinetic energy, $k T$. At intermediate temperatures $(\log T \approx 5$ ) and densities of $\log \rho \approx 0$, temperature and pressure ionization are of comparable importance. In this regime, thermal excitation of hydrogen atoms is significant and they are immersed in a moderately coupled plasma $(\Gamma \approx 1)$ where electrons are partially degenerate $(\theta \approx 1)$. This regime is particularly difficult to treat as the internal levels of the atoms are strongly perturbed by the surrounding plasma. For most elements, this is, along with pressure ionization, the regime where equations of state are most unreliable.

At low densities and high temperatures, radiation pressure $P_{\text {rad }}$ becomes larger than the gas pressure, $P_{\text {gas }}$. Finally, the upper left part of Fig. 1 represents conditions which are not realized in astrophysical contexts, where hydrogen is a high- $T$ molecular solid or possibly forms a Coulomb lattice.

Interior models of various hydrogen-rich objects are shown by dotted curves in the density-temperature plane of Fig. 1. The gaseous envelope of Jupiter is shown by the curve labeled 'a.' The envelope is dominated by molecular hydrogen and it goes through the region of pressure ionization. If the PPT calculated by Saumon and Chabrier (1992) occurs in nature, it should also be found in the envelope of Jupiter. Just below the PPT, the dense molecular fluid becomes strongly non-ideal due to the strongly repulsive intermolecular forces.

Curves ' $b$ ', ' $c$ ', and 'e' represent main sequence stars with masses of 0.3 , 1 and $15 M_{\odot}$, respectively, where $M_{\odot}$ is the mass of the Sun. The $15 M_{\odot}$ star has the simplest EOS physics. It is fully ionized throughout its interior and the plasma is very weakly coupled $(\Gamma<<1)$. Electron degeneracy is also weak $(\theta<<1)$. The contribution of radiation pressure is significant, however and the ratio $P_{\text {rad }} / P_{\text {gas }}$ is roughly constant in the interior. The solar model (c) is both cooler and denser. Accurate modeling requires attention to relatively weak non-ideal effects $(\Gamma \approx 0.1)$ and partial electron degeneracy near the center. Recombination of the plasma into $\mathrm{H}$ atoms affects the structure near the surface. Low-mass stars, such as the $0.3 M_{\odot}$ model shown, probe more complex areas of the phase diagram. Electrons are partially degenerate throughout most of the star and the electrostatic interactions in the plasma become significant. The model crosses the difficult regime where $\Gamma$ and $\theta$ are of order unity. In the outer part of the model, recombination forms atoms in a non-ideal regime and finally molecules form at the very surface.

The curve labeled ' $d$ ' is a $12500 \mathrm{~K} \mathrm{DA}$ white dwarf envelope, stratified into hydrogen-rich and helium-rich layers surrounding a carbon core $(\mathrm{H}, \mathrm{He}$ and $\mathrm{C}$, respectively). Only the outermost layer, consisting of pure hydrogen, is 
shown here. In this layer, hydrogen forms a weakly coupled, non-degenerate plasma. Atomic hydrogen is found at the very surface of the star. The relatively low densities and high temperatures characteristic of this layer indicate that non-ideal effects in the EOS are of moderate importance.

\subsection{The Free Energy Minimization method}

Nearly all EOS involving chemical equilibrium, i.e. molecular dissociation or ionization of atoms and ions, are based on the free energy minimization technique (FMIN). One major exception is the EOS of Rogers (1981) which is based on an activity expansion. It is briefly discussed in $\S 4$.

The FMIN method is well described in Graboske, Harwood and Rogers (1969 and references therein), Fontaine, Graboske and Van Horn (1977) and Hummer and Mihalas (1988). The approach is particularly simple. Given a mathematical model for the Helmholtz free energy of the system as a function of total volume, temperature and particle numbers, $F\left(V, T,\left\{N_{i}\right\}\right)$, the chemical equilibrium of the mixture is obtained by minimizing of $F$ at fixed $V$ and $T$, subject to the stoichiometric constraints imposed by the chemical reactions taking place in the system. This fixes the $\left\{N_{i}\right\}$, and the pressure and entropy can then be calculated by differentiation of the free energy with respect to $V$ and $T$, respectively, at fixed $\left\{N_{i}\right\}$. These so-called first derivatives of the free energy are given by:

$$
P=-\left.\frac{\partial F}{\partial V}\right|_{T,\left\{N_{i}\right\}} \quad \text { and } \quad S=-\left.\frac{\partial F}{\partial T}\right|_{V,\left\{N_{i}\right\}} .
$$

The specific heats, compressibility, thermal expansion coefficients, adiabatic gradients are obtained by further differentiating $P$ and $S$ with respect to $V$ and $T$ and are second derivatives of the free energy. Differentiation amplifies the features and defects in $F$ and since the second derivatives are usually obtained numerically, they are also prone to numerical noise.

The FMIN method becomes truly useful when the grand partition function of the system $\mathcal{Z}$ is written as the product of kinetic, internal and configuration contributions:

$$
\mathcal{Z}=Z_{\text {kin }} Z_{\text {int }} Z_{\text {cont }} \text {. }
$$

Fontaine, Graboske and Van Horn (1977) give an excellent discussion of the four approximations leading to this factorizability. In practice, small 
deviations from exact factorizability are usually accommodated by corrections based on expansions in terms of a small parameter. However, when the particles of the system interact strongly, the spectrum of bound states is affected and $Z_{\text {int }}$ and $Z_{\text {conf }}$ are not factorizable anymore. Similarly, the bound state configuration determines the interaction potentials and modifies $Z_{\text {conf }}$. The total partition function is nevertheless factorized, with some modification of the spectrum of bound states entering the internal partition function $Z_{\text {int }}$ (IPF) based on the interaction potentials. A great variety of treatments of this problem have been used, some very crude and with very low internal consistency, some quite sophisticated. However, there is no formally exact treatment and this is the source of many disagreements between EOS computed with this method.

Despite this shortcoming, the FMIN technique has several powerful advantages. In principle, it ensures thermodynamic consistency of the resulting equation of state. All the physics and approximations appear at the outset in the free energy model and are therefore quite visible. No additional approximations are required. Contrary to expansion techniques, contributions with strongly non-linear dependence on density or temperature can be included with no additional effort. Under the assumption of factorizability, the free energy model becomes a sum of terms, each involving a different physical contribution. This is extremely convenient, as each term becomes a subroutine in the EOS code. Terms can be added, removed and modified with great ease. Hummer and Mihalas (1988) point out that this method will work for any free energy model. However, because the validity of the resulting EOS is hard to test, unless it violates fundamental constraints or known limits, great care must be taken in constructing the free energy model to ensure internal consistency.

\subsection{Choice of equations of state}

In the spirit of clarity and conciseness, the comparison is limited to four hydrogen equations of state: the table of Fontaine, Graboske and Van Horn (1977), the pure $\mathrm{H}$ case of the H/He EOS of Magni and Mazzitelli (1979), $\dagger$ a pure hydrogen calculation based on the model developed by Mihalas, Hummer and Däppen (Däppen et al. 1988 and references therein), and the EOS of Saumon and Chabrier $(1991,1992)$. Hereafter, these four equations of state will be referred to as FGVH, MM, MHD and SC, respectively.

These EOS span over 15 years of effort in developing reliable EOS for

$\dagger$ A study of the MM EOS table which we obtained in 1987 shows that it is much improved over the version published in Magni and Mazzitelli (1979). 


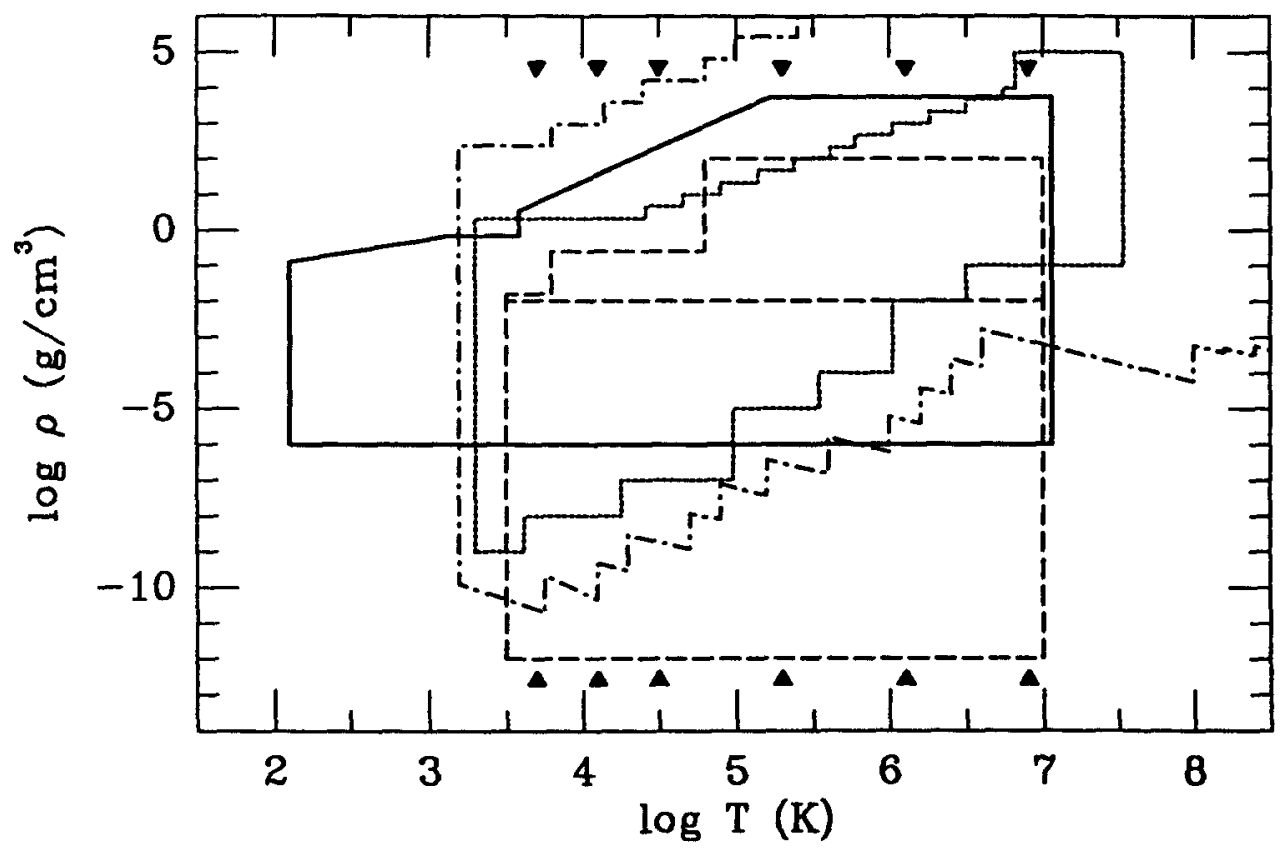

Fig. 14.2 Density-temperature domain covered by the four EOS considered. MHD, dashed line (note the extension above $\log \rho=-2$ ); FGVH, dotted line; SC, solid line; MM, dot-dashed line. The MM EOS extends beyond the limits of the figure. Pairs of triangles show the isotherms selected for the EOS comparison.

stellar envelopes and interiors and they are representative of the better EOS currently in use by the community. Except for the SC EOS, they have been used extensively in a variety of astrophysical contexts. All four equations of state are based on the FMIN technique. While they have a number of features in common, they differ greatly in detail, in the level of internal consistency of the model and in the accuracy of the various contributions to the free energy.

The $(\rho, T)$ domain covered by each EOS is shown in Fig. 2. Two contiguous domains are shown for the MHD EOS. While the free energy model was developed for $\log \rho \leq-2$, the calculation was pushed to higher densities, as shown by the figures in Mihalas, Däppen and Hummer (1988) and in Däppen et al. (1988). This "extension" of the MHD EOS above $\log \rho=-2$ is shown in Fig. 2 and is discussed further in $\S 6$.

Additional comparisons between the SC EOS and other equations of state used in astrophysical problems have also been performed. The $\mathrm{H} / \mathrm{He} \mathrm{EOS}$ of Marley and Hubbard (1988) was developed for modeling the interior of giant planets. It shares many similarities with the model of SC and under 
the low-temperature conditions relevant to giant planets $(\log T<4)$, the two EOS are nearly identical. Differences arise in a narrow density domain centered on pressure ionization, where Marley and Hubbard simply interpolated between the dense molecular fluid and the fully pressure-ionized plasma. For $\log T>4$, their approximate treatment of temperature ionization leads to substantial disagreement. A more detailed discussion is given in Chabrier et al. (1992).

The hydrogen EOS developed at the Los Alamos National Laboratory, known as the SESAME 5251 EOS, was developed by Kerley (1972). A comparison of pressures $P$ and internal energies $U$ (second derivatives are not directly available) from an EOS table obtained in 1984 with the SC EOS shows a relatively good agreement for $\log T \geq 3.7$. This is somewhat surprising if we consider that the SESAME 5251 hydrogen EOS is actually a deuterium EOS scaled in density. Differences in pressure reach a maximum of $25 \%$ in the regime of pressure ionization. In the regime of temperature ionization differences are as high as $40 \%$. At the lower temperatures where molecules dominate the EOS, systematic differences of $\approx 6 \%$ are found in $U$ (Saumon and Van Horn 1987). This arises from the density scaling procedure which is not appropriate in the molecular phase. The energy levels of the molecule depends on the moment of inertia and the reduced mass of $\mathrm{D}_{2}$ which are twice as large as for $\mathrm{H}_{2}$.

Rogers (1981) has developed an EOS with an approach entirely different from FMIN, using an activity expansion which considers only protons and electrons interacting with the Coulomb potential. Bound states (atoms) arise naturally in this approach and are not treated as a separate chemical species, as in the FMIN method. This approach is very rigorous and fundamental (Rogers 1994). Over the $(\rho, T)$ domain where this complex method can be presently solved, it leads to a most accurate EOS. While we have not yet compared it with the SC EOS, it has been compared with the MHD EOS under the conditions found in the solar envelope (see Däppen 1994 and Rogers 1994). The two equations of state are in extremely good agreement, with differences of less than $0.1 \%$ in the second derivatives of the free energy. While such differences are important when comparing the computed solar oscillation spectrum to the wealth of extremely precise data, they are completely negligible in all other astrophysical situations. It is very satisfying that two equations of state based on entirely different approaches should agree so well. This indicates that our understanding of the EOS of normal stellar material is now excellent, at least over some parts of the phase diagram. 


\subsection{About the Saumon-Chabrier EOS}

The free energy model underlying the SC EOS is described in details in Saumon and Chabrier $(1991,1992)$ but the EOS itself is not yet available (Saumon, Chabrier and Van Horn 1993). The model is summarized in the review by Chabrier (1994) and a few additional remarks relevant to the present comparison follow.

Pressure ionization received particular attention in the SC EOS calculation. It was found that the adopted free energy model (and a number of variants) becomes thermodynamically unstable and predicts the existence of a first order phase transition between a mostly molecular phase and a dense, partially ionized phase. This plasma phase transition (PPT) is shown in Fig. 1. It terminates at high temperature at a critical point located at $T_{c}=15300 \mathrm{~K}, P_{c}=6.14 \times 10^{11} \mathrm{dyn} / \mathrm{cm}^{2}$, and $\rho_{c}=0.35 \mathrm{~g} / \mathrm{cm}^{3}$. Pressure ionization is a most difficult problem in EOS calculations and much remains to be said on this challenging topic. There is currently no experimental result which bears on the existence of the PPT.

To allow for the possibility that the PPT is not realized in nature, there is an "interpolated" version of the SC EOS where the discontinuities associated with the PPT have been smoothed by interpolation. It is otherwise identical to the SC EOS with PPT. This interpolated version used for the comparisons in $\S 6$. The interpolated region has an irregular shape but it extends roughly over $3.50<\log T<4.78$ and $-0.5<\log \rho<0.5$. Ideally, the interpolation of $P$ and the entropy $S$ (or $P$ and $U$ ) should be constrained by the requirement of thermodynamic consistency, which reflects that $P$ and $S$ are not independent quantities but derive from the same thermodynamic potential, in this case the Helmholtz free energy. Fontaine, Graboske and Van Horn (1977) applied this constraint when interpolating across the regime of pressure ionization. In the case of the SC EOS, however, it was found that the requirements of 1) continuity of $P, S$ and their derivatives at the boundaries of the interpolation region and of 2) thermodynamic consistency overconstrain the interpolation. This difficulty can be avoided by widening the density range of the interpolation but only to an unacceptable degree where parts of the EOS table believed to be reliable (based on experimental data and an assessment of the model) would be replaced by less accurate, interpolated values. This suggests that while there may not be a PPT in hydrogen, pressure ionization probably occurs rather suddenly. Reliable EOS values were preserved at the cost of losing thermodynamic consistency and $P$ and $S$ were interpolated separately along isotherms over as narrow a density range as possible. 
The free energy model underlying the SC EOS can be improved upon and ameliorations are being considered. The most important of which involves the effect of charged particles on the bound states of hydrogen atoms. Interactions with neighboring particles, charged and neutral, affect the number of bound states, or the internal partition function, of atoms and molecules. A proper treatment of this effect is essential for an accurate description of partial dissociation and ionization, particularly at larger densities $(\log \rho \geq-3)$. While the activity expansion of Rogers (1981) accounts for this naturally and rigorously, the FMIN method is only weakly constrained in this respect.

In its current form, the free energy model developed by Saumon and Chabrier (1992) accounts only for the effect of neutral particles on bound states (by an excluded volume effect). In reality, neighboring charged particles also affect the bound states by inelastic collisions with bound electrons and also through the fluctuation micro-electric field induced by their thermal motion. This microfield has the effect of a time dependent perturbation on the Coulomb potential of the nucleus and can induce Stark ionization of the upper levels of an atom. Collisions and microfield effects on hydrogenic atoms are discussed in great details in Hummer and Mihalas (1988) who conclude that for $\log \rho \leq-1.5$, the microfield is the dominant mechanism. Being caused by random thermal motions, the fluctuating microfield is described by a statistical distribution. Hummer and Mihalas have adopted the $\Gamma=0$ Holtzmark distribution. We have found that this distribution, which does not account for the correlations which arise between charged particles at $\Gamma>0$, has much too strong an effect on the IPF and leads to spurious results for $\Gamma \approx 1$. Generating microfield distributions for finite $\Gamma$ is computationally involved and a suitable, parametrized form was not available when the SC EOS was computed. As a consequence, the effect of the microfield is ignored altogether until an adequate distribution function becomes available. The net effect of this omission is that as the gas becomes mostly ionized by temperature, the IPF is less affected by the neighboring particles than when it was surrounded by neutral particles. This creates a long tail of residual atoms in the partial ionization zone. We will return to this point in $\S 6$.

Two thermodynamic surfaces from the SC EOS are show in Figs. 3 and 4. Only second derivatives of the free energy are shown because they display the various physical regimes more clearly than first derivatives such as $P$ and $S$. They also amplify defects in $F$ they are very useful to reveal flaws in an EOS as well as its degree of smoothness. Note that the MHD free energy model is sufficiently simple to allow for analytic differentiation of $F$ and their EOS is consequently very smooth. As emphasized in the reviews 


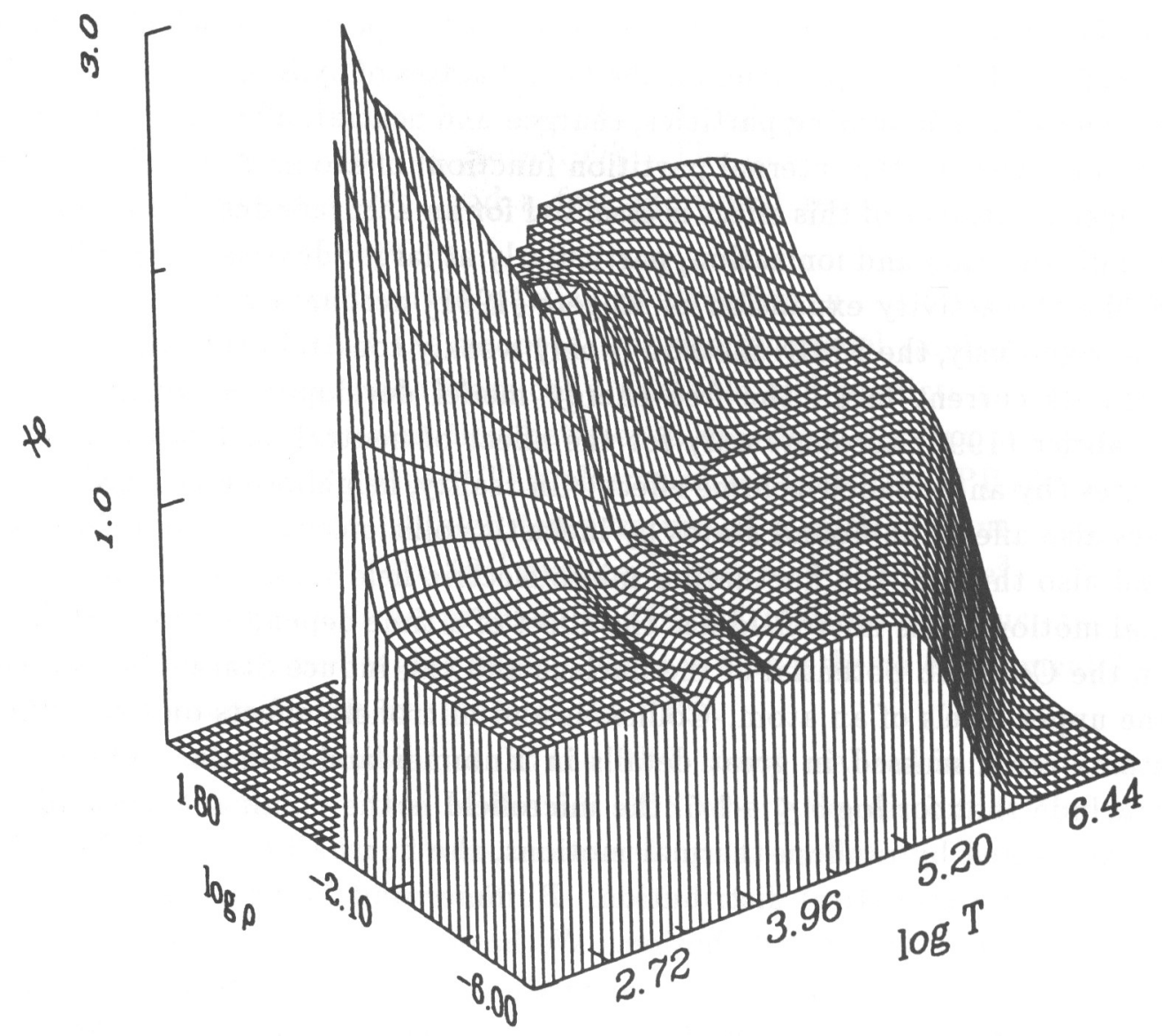

Fig. 14.3 Inverse compressibility, $\chi_{\rho}=\partial \log P /\left.\partial \log \rho\right|_{T}$, for the $(\rho, T)$ range covered by the Saumon-Chabrier EOS (interpolated version). The $(\rho, T)$ grid shown is that of the original tabular data and no smoothing has been applied.

by Fontaine (1994) and Däppen (1994), smoothness of the EOS can be more desirable than accuracy in the context of non-adiabatic stellar pulsations.

Figure 3 shows $\chi_{\rho}=\partial \log P /\left.\partial \log \rho\right|_{T}$, which measures the stiffness of the EOS. For an ideal gas, $\chi_{\rho}=1$, as can be seen over most of the lowdensity part of the figure. The photon gas pressure depends only on $T$ and $\chi_{\rho}=0$ in that limit. The degenerate electron gas is less compressible than the Maxwell-Boltzmann ideal gas and $\chi_{\rho}$ rises to a plateau at 5/3. The two shallow "valleys" seen on either side of $\log T=4$ are due to molecular dissociation and ionization. They clearly separate the regions dominated by $\mathrm{H}_{2}, \mathrm{H}$ and $\mathrm{H}^{+}$, respectively. The very steep rise seen at low- $T$ and high$\rho$ is caused by the repulsive core of the $\mathrm{H}_{2}-\mathrm{H}_{2}$ interaction potential. Like 


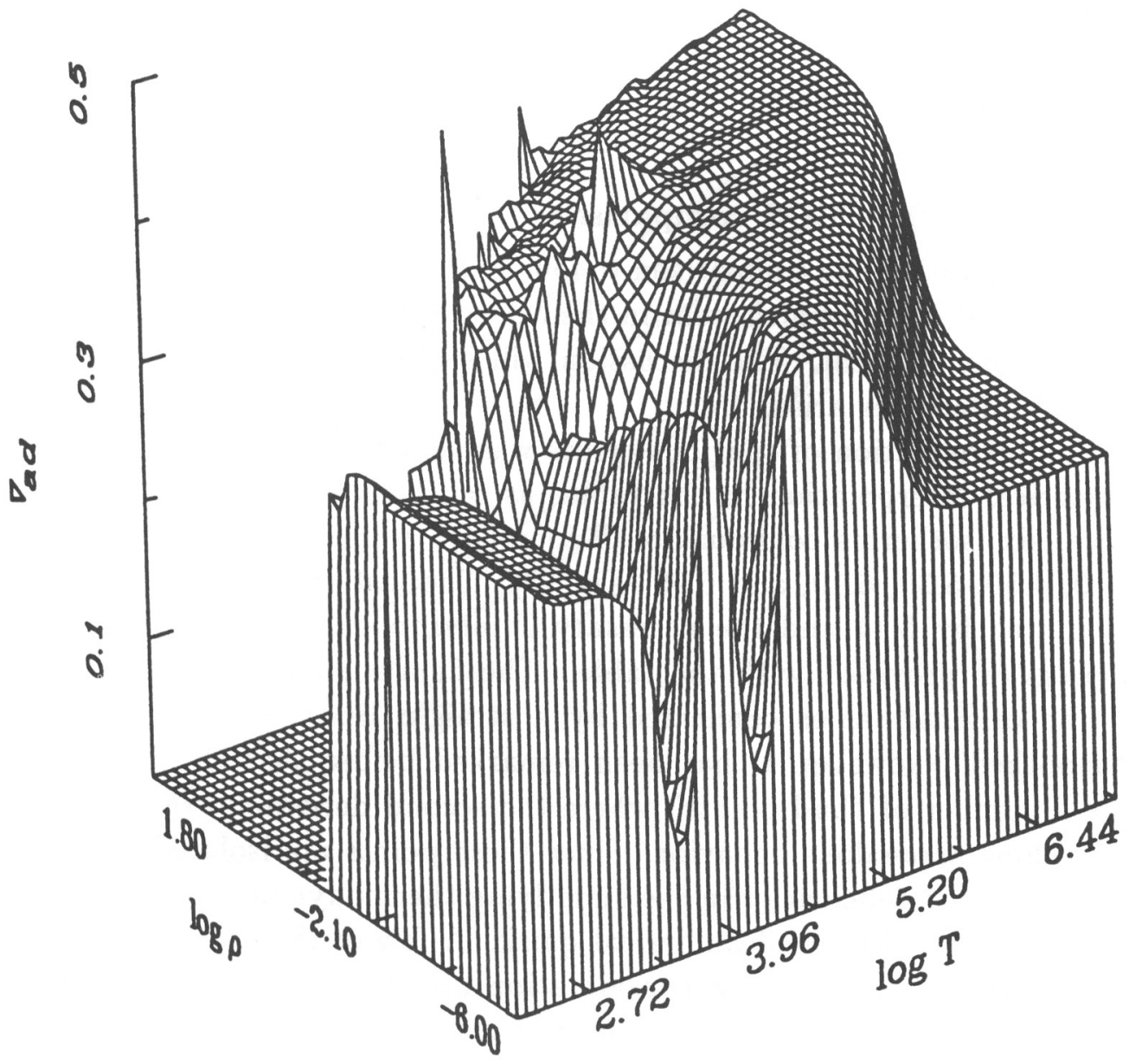

Fig. 14.4 Adiabatic gradient, $\nabla_{\mathrm{od}}=\partial \log T /\left.\partial \log P\right|_{S}$ for the $(\rho, T)$ range covered by the Saumon-Chabrier EOS (interpolated version). The $(\rho, T)$ grid shown is that of the original tabular data and no smoothing has been applied.

all liquids, dense fluid $\mathrm{H}_{2}$ is relatively incompressible, a property reflected by the high value of $\chi_{\rho}$. There are a few spurious features caused by the interpolation procedure at intermediate $T$ and high $\rho$. As indicated in Fig. 2, the EOS does not extend to the low- $T$ and high- $\rho$ limit where $\chi_{\rho}=0$. Figure 3 is best interpreted in reference to the phase diagram shown in Fig. 1.

Most of the physical regimes discussed above can be identified in Fig. 4 which shows the adiabatic temperature gradient, $\nabla_{\mathbf{a d}}=\partial \log T /\left.\partial \log P\right|_{S}$. When expressed in terms of $\rho$ and $T, \nabla_{a d}$ is a function of the four second derivatives of $F: \partial P /\left.\partial \rho\right|_{T}, \partial P /\left.\partial T\right|_{\rho}, \partial S /\left.\partial \rho\right|_{T}$ and $\partial S /\left.\partial T\right|_{\rho}$. It therefore combines all the defects and noise found in the second derivatives. This is 
the origin of the several spikes seen in the interpolation region. Other peaks and oscillations found along the high- $\rho$ border of the EOS are caused by edge effects in the table.

\subsection{The EOS comparison}

Even by limiting the comparison to four EOS, it is not possible to do justice to the great efforts which went in their development or to review the merits of each one of them. The discussion will be limited to the areas were the largest differences arise.

The introduction of spurious errors was kept to a minimum by avoiding numerical interpolation in the tables as much as possible. For that purpose, six isotherms common to all four EOS were selected: $\log T=3.70$, $4.10,4.50,5.30,6.10$, and 6.90 (Fig. 2). The last three isotherms are not tabulated by $M M$ and the necessary $T$-interpolation was performed with a program provided with the table. The figures show the density points of the original tables connected by a straight line. The EOS were not "smoothed", however, it has been remarked before that the FGVH EOS has a number of "bad points" where the second derivatives of the free energy show anomalous behavior. Since these points are isolated, they must not arise from deficiencies in the underlying thermodynamic description but represent some localized numerical quirk. A few of these points are found in the six isotherms under consideration and the discordant values were corrected by a simple interpolation in density. These points are located at $(\log T, \log \rho):(4.10,-5.667),(4.50,-3.667),(5.30,-2.333)$ for $\nabla_{\text {ad }}$.

The quantities compared are $\log P, \log U$ and $\nabla_{\mathbf{a d}}$ for all six isotherms. Exceptions are the MHD EOS which is not shown for $\log T=6.10$ and 6.90 , the table available being limited to $\log T \leq 6$, and the MM EOS which gives only $\log P, \nabla_{\text {ad }}$ and $C_{p}$, the specific heat. This last quantity is not used in the present comparison. Note that all quantities shown here are taken directly from the EOS tables and are not constructed from other quantities by using thermodynamic identities, for example. This avoids introducing potential errors due to thermodynamic inconsistency in the EOS or numerical inaccuracies in the procedure. In all four cases, the zero of energy is chosen as the ground state of the $\mathrm{H}_{2}$ molecule and the contribution of the photon gas is included.

The four EOS are compared in Figures 5-9 where it is readily apparent that the differences can be substantial. As a point of reference, the SC EOS indicates that $P$ and $U$ are within $1 \%$ of their ideal gas value for $\log \rho<-2$. It is easy to verify that the non-ideal terms are very small at this density 


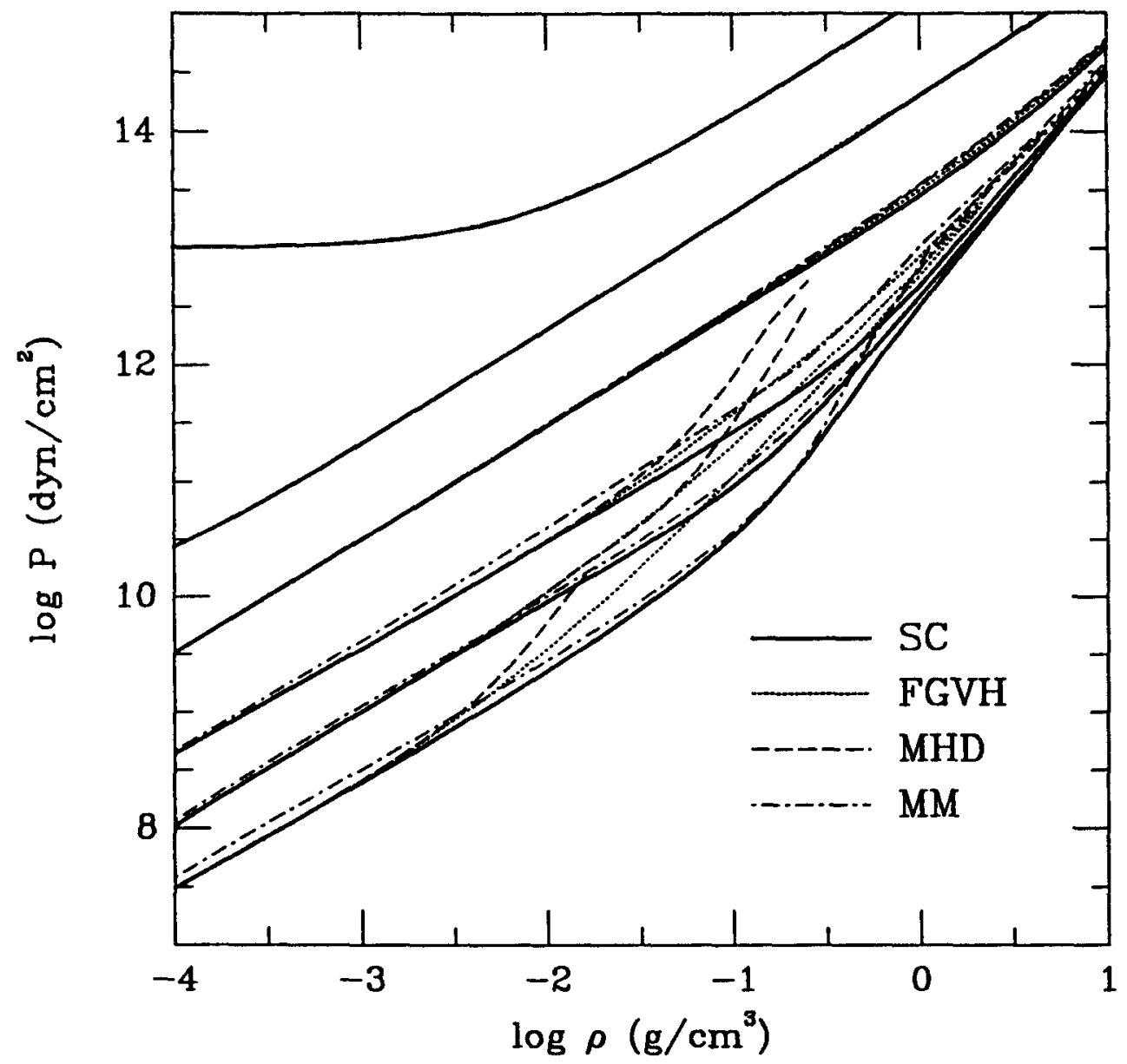

Fig. 14.5 Comparison of pressure isotherms from the four equations of state. The isotherms are (from top to bottom): $\log T=6.90,6.10,5.30$, $4.50,4.10$ and 3.70 .

by comparing the volume occupied by atoms (or molecules) to the total volume. Surprisingly, significant differences are found even for densities below $\log \rho=-2$.

\subsubsection{The pressure}

Figure 5 shows the pressure from the four EOS along the six isotherms. At lower and at higher densities than shown in this figure, the agreement is satisfactory. The two hottest isotherms correspond to a fully ionized gas of $\mathrm{H}^{+}$and $e$ interacting weakly in the Debye-Hückel limit. The photon pressure dominates gas pressure when $P$ becomes independent of $\rho$ along the $\log T=6.90$ curve. The agreement is excellent in this relatively simple 
regime but severe divergences are found at lower temperatures. Curiously, the MM EOS systematically overestimates $P$ at low densities, where the gas is ideal for all practical purposes. This is most likely due to an overestimate of the degree of dissociation and perhaps ionization which arises from their treatment of the IPF of $\mathrm{H}$ and of $\mathrm{H}_{2}$. Their IPF for $\mathrm{H}_{2}$ has been corrected since we obtained the MM table in 1987 (Mazzitelli 1993).

For the three lowest isotherms, the MHD and FGVH EOS predict much higher pressures than either the SC or the MM EOS at moderate densities. For temperatures up to $\log T=3.6$, the SC EOS reproduces experimental results and can be considered as a reference for this comparison. The high pressures of FGVH and MHD are caused by the hard sphere potential used to model the interactions between neutral particles. This potential qualitatively models the strongly repulsive cores of the actual potentials, but being infinitely repulsive, it fails to describe the softness of the repulsion. This feature of neutral-neutral interactions becomes important at high densities. The hard sphere potential is too repulsive at high densities and leads to overestimated pressures even in a regime where the gas should be nearly ideal. The authors of the MHD EOS point out that their EOS should be used for $\log \rho<-2$, a safe limit at low- $T$ in view of the above observation.

In the regime of pressure ionization $(-0.5<\log \rho<0.5)$, the SC, MM and FGVH EOS can differ by up to a factor of 2 . In all three cases, thermodynamic quantities were smoothly interpolated between a low-density and a high-density regime where the authors felt that their respective EOS were reliable.

\subsubsection{The internal energy}

Most of the features discussed above can also be seen in the internal energy $U$, shown in Fig. 6. Again, we see that for $\log T<4.50$ and $\log \rho>-2$, the hard sphere model used by FGVH and MHD leads to an overestimate of $U$. The $\log \rho=-2$ limit recommended by MHD is a sensible choice for $U$ as well as for $P$.

The two intermediate isotherms illustrate the importance of a careful treatment of the influence of neighboring particles on the IPF. For the $\log T=4.50$ isotherm, SC lies above MHD and the reverse is true for $\log T=5.30$. At these low densities, characteristic of the ideal gas, this arises from differences in the degree of ionization, which is directly affected by the IPF of atomic hydrogen. At these temperatures, thermal excitation of $\mathrm{H}$ becomes significant and the chemical equilibrium depends on how 


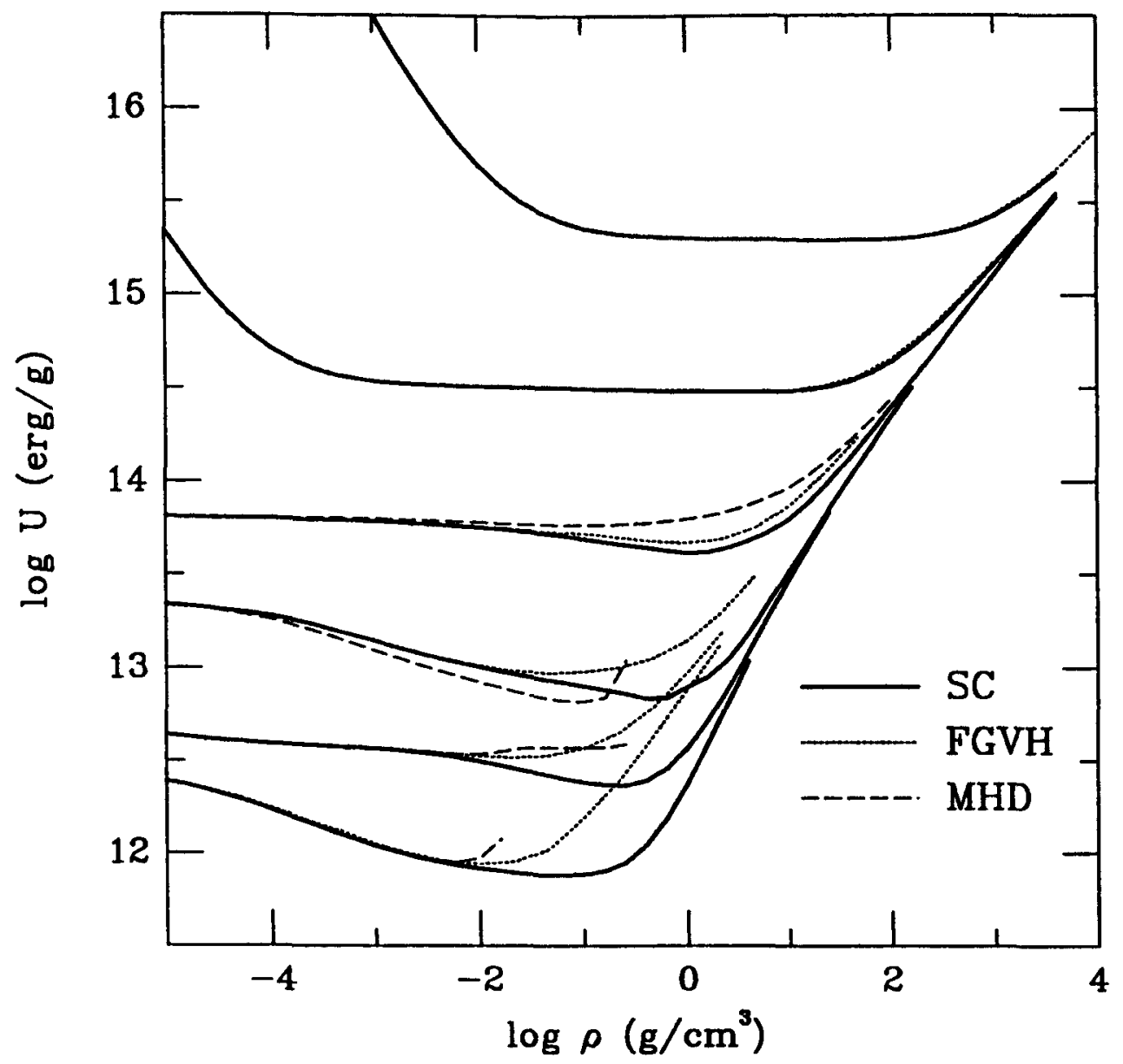

Fig. 14.6 Comparison of internal energy isotherms. 'The isotherms are (from top to bottom): $\log T=6.90,6.10,5.30,4.50,4.10$ and 3.70 .

many states are allowed in the IPF sum. $\dagger$ This effect was not visible in the pressure because it is relatively insensitive to excitation energies of bound species.

At $\log T=4.50$, the degree of ionization is sufficiently low for the finite "size" of atoms to be the main non-ideal contribution. This, in effect, is an excluded volume interaction which removes the upper levels of the IPF to ensure that the atoms do not "overlap." MHD adopted a fixed and somewhat arbitrary diameter for the $\mathrm{H}$ atom in its ground state (1.06 $\AA$ ) while SC use a thermodynamic criterion (Saumon and Chabrier 1991) to compute a temperature and density dependent value ranging from 1.1 to

† The SC and MHD EOS do not use a cut off in the IPF sum but a gradual removal of bound states based on the occu pation probability formalism presented in Hummer and Mihalas (1988). It is nevertheless useful to thin $k$ in terms of a sharp cut off in the present context. 
about 1.6 $\AA$. Fewer states are retained in the IPF when the hard sphere diameter is larger, favoring a higher degree of ionization in the SC EOS and a larger $U$. Because the SC EOS uses more realistic interaction potentials between neutral particles and a thermodynamic criterion to obtain the hard sphere diameters of $\mathrm{H}$ and $\mathrm{H}_{2}$, it is more reliable in this regime than the MHD EOS.

The situation is quite different along the $\log T=5.30$ isotherm where the degree of ionization is high and atoms are surrounded mostly by charged particles. As discussed in $\S 5$, the motion of the ions and electrons induces a fluctuating micro-electric field which can cause Stark ionization of the upper levels of the atom, thereby removing them from the IPF. Since this effect is missing in the SC EOS, the IPF retains too many states and the degree of ionization as well as the internal energy are underestimated. According to Fig. 6, this effect is not very large, but the MHD EOS is nevertheless more accurate in this regime.

Along the $\log T=5.30$ isotherm, the MHD and the SC EOS differ most notably for $-1<\log \rho<1$. Under these conditions, pressure ionization occurs where thermal excitation of the atoms is large. We discussed this regime in $\S 2$. While the MHD model is well beyond its limit of validity $(\log \rho \leq-2)$, none of the EOS presented here can be considered reliable in this difficult regime.

\subsubsection{The adiabatic gradient}

As discussed above, the second derivatives of the free energy are very sensitive to the choice of thermodynamic model and display its flaws prominently. The adiabatic temperature gradient is particularly interesting since it forms the basis of the Schwarzschild criterion for convective instability in stars. The six isotherms for the adiabatic gradient are shown on Figs. 7-9. Except in a few well known limits, figures of $\nabla_{\text {ad }}$ are particularly difficult to interpret physically. We will limit the analysis to listing the failures and problems with each EOS.

Figure 7 shows the two lower isotherms over a wide density range. The overall wavy structure is caused by partial dissociation and ionization. A number of features are immediately apparent:

- Even at very low densities where the gas is ideal, the agreement is not perfect. Differences of $10 \%$ are commonplace.

- The FGVH EOS can be very noisy.

- The MHD EOS shows pathological behavior for $\log \rho>-2$, once again reinforcing their warning about not using their EOS above this limit. 


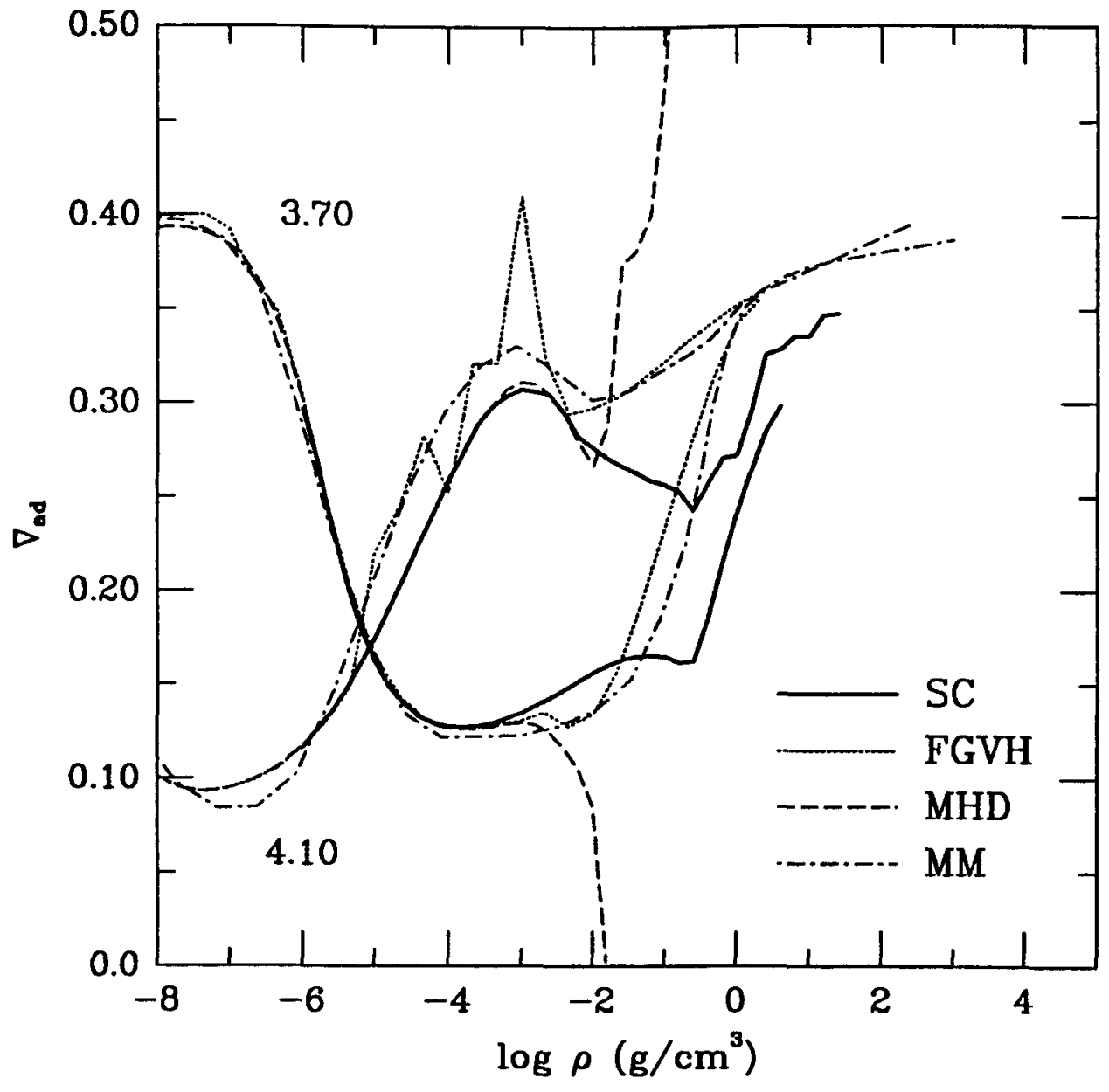

Fig. 14.7 Comparison of adiabatic gradient isotherms showing the $\log T=$ 3.70 and 4.10 isotherms.

- The SC EOS is not very smooth in the regime of the fully ionized plasma $(\log \rho>0.5)$.

The next two isotherms are displayed on Fig. 8. Again, the wavy structure seen for $\log T=4.50$ is due to partial ionization. Hydrogen is nearly fully ionized everywhere along the $\log T=5.30$ isotherm and the drop to $\nabla_{\mathrm{ad}}=0.25$ at very low densities is due to the photon gas. We find that:

- There are still differences in the ideal gas regime, but they are below the $10 \%$ level.

- The FGVH EOS appears smoother in this regime

- Above $\log \rho=-1.5$, the MHD EOS shows pathological behavior along both isotherms. 


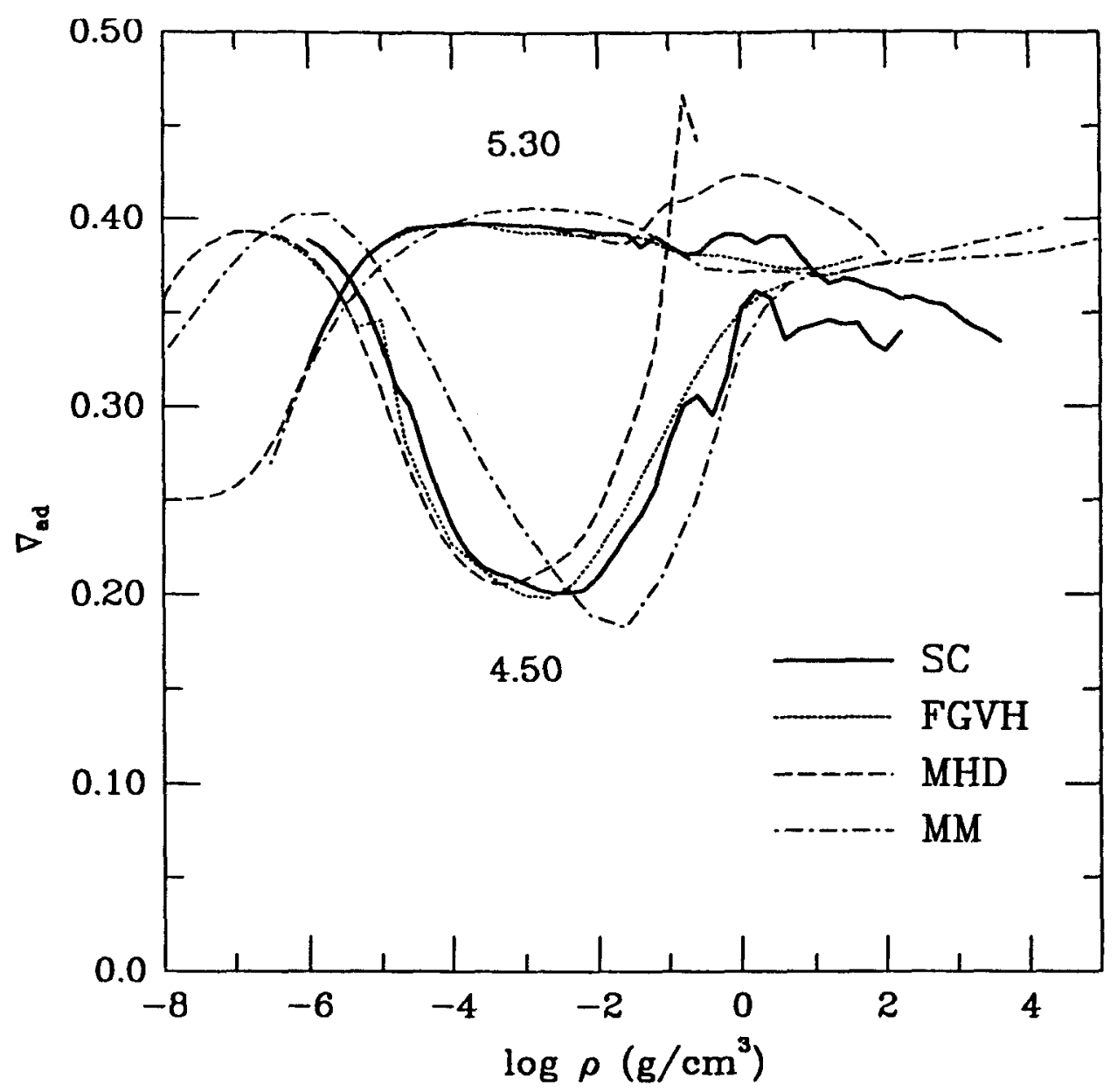

Fig. 14.8 Comparison of adiabatic gradient isotherms showing the $\log T=$ 4.50 and 5.30 isotherms.

- For the $\log T=4.50$ isotherm, the MM EOS shows a "phase lag." This indicates an ionization zone which is displaced to comparatively higher densities. This originates in their treatment of the IPF.

- At the high-density end of these isotherms, $\Gamma>10$ and $\theta<1$, conditions under which the Coulomb interactions are strong. In FGVH and MM, these are described with a Thomas-Fermi-Dirac model and both show $\nabla_{\text {ad }}$ rising as the density is increased. On the other hand, SC use a screened onecomponent plasma model (SOCP, Chabrier 1994), a much more accurate description of the plasma, and find that $\nabla_{\text {ad }}$ decreases along the isotherm. The SC EOS remains rather noisy in this regime.

Finally, Figure 9 shows the two hottest isotherms. For a pure photon gas, $\nabla_{\text {ad }}=0.25$ and it approaches 0.4 , the value for a non-interacting (ideal), classical, monoatomic gas, as the pressure of the plasma comes into play. 


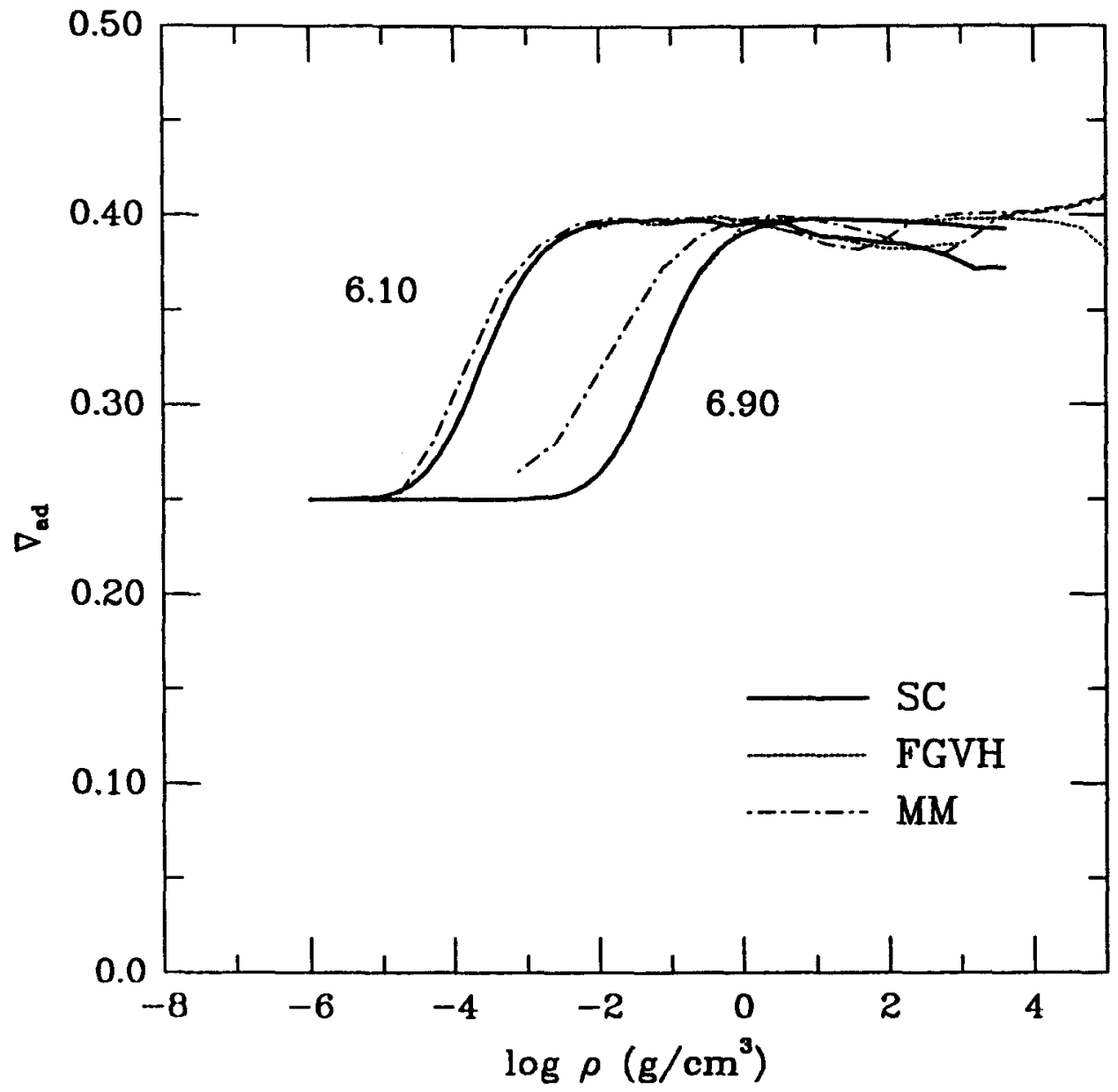

Fig. 14.9 Comparison of adiabatic gradient isotherms showing the $\log T=$ 6.10 and 6.90 isotherms.

Both of these limits are readily apparent on this figure. The adiabatic gradient of a mixture of photons and non-interacting protons and electrons can be calculated analytically (Cox and Giuli 1968, $\S 9.17$ ), a result accurately reproduced by FGVH and SC. The divergence of the MM curves from the analytic expression cannot be explained on physical grounds. At high densities, $\nabla_{\text {ad }}$ drops below 0.4 due to relatively weak to moderate Coulomb interactions $(\Gamma<1)$. Both the FGVH and SC EOS show a downward trend in $\nabla_{\mathrm{ad}}$ and agree quite well while the MM EOS displays an increase similar to that observed in Fig. 8.

Because of its strong connection with convective instability, $\nabla_{\text {ad }}$ plays an important role in models of stellar interiors and envelopes. Figures 7-9 show differences of the order of $10 \%$ in the ideal gas regime of partial dissociation and ionization, underscoring the sensitivity of $\nabla_{\mathrm{ad}}$ to the treatment of the 
states in the IPF. When strong non-ideal effects come into play, it appears that the adiabatic gradient remains a rather poorly determined quantity.

\subsection{Concluding remarks}

This exercise of comparing several equations of state developed for applications to astrophysical problems (mainly stellar interiors) reveals that the situation is not as satisfactory as is commonly assumed. Much progress has been accomplished over the time span represented by these EOS: the SC and the MHD EOS represent considerable improvement over the older FGVH and MM EOS. This is due in part to new high-pressure experiments which probe the $\mathrm{H}_{2}-\mathrm{H}_{2}$ potential to smaller interparticle separations, the development of a solid knowledge of dense plasmas through numerical simulation and a more acute awareness of the importance of consistency between the treatment of the internal partition function and the interactions between particles.

It also shows that each of these EOS has flaws or limitations, most of which can be addressed in the near future. The most challenging areas remain associated with partial dissociation and ionization for hydrogen. The treatment of temperature ionization with the FMIN method has improved considerably in the last few years but we have seen that none of the EOS presented here is truly satisfactory in this respect. The more rigorous activity expansion technique may provide a definitive treatment of temperature ionization. On the other hand, pressure ionization remains by far the most poorly understood phenomenon and maintains a shroud of uncertainty over a part of the phase diagram which is important for low-mass stars, brown dwarfs and most critically, the jovian planets.

The calculation of such equations of state is a complex problem. Those presented here each required a few man-years of effort, and still they display flaws and problems of various importance. This raises strong doubts about the validity of the much simpler and often crude equations of state used in many astrophysical problems. Any problem calling for an equation of state should first be cast in a phase diagram (Fig. 1) to determine the relevant physical regimes, the magnitude of non-ideal effects and whether partial ionization and dissociation are expected. In numerous cases, the EOS is sufficiently simple for semi-analytic treatments to be adequate. However, if non-ideal effects are expected and the accuracy of the final result is important, there is no justification for not using the appropriate tabular equation of state. In conclusion, astrophysicists should be more critical of the equations of state they use. 
I am very grateful to F. D'Antona, and to D. G. Hummer, who kindly provided the MM and the pure hydrogen MHD EOS tables, respectively. I thank G. Fontaine who generated Figs. 3 and 4 and provided the white dwarf model shown in Fig. 1, and F. J. Swenson for sending me the $0.3 M_{\odot}$ main sequence star model (Fig. 1.). This research was supported in part by NSF grant AST-8910780 and by NASA grant HF-1051.01-93A from the Space Telescope Science Institute, which is operated by the Association of Universities for Research in Astronomy, Inc., under NASA contract NAS526555.

\section{References}

Chabrier, G., these proceedings (1994)

Chabrier, G., Saumon, D., Hubbard, W.B., and Lunine, J. Ap. J., 391,817 (1992)

Clayton, D. D., Principles of Stellar Evolution and Nucleosynthesis, $2^{\text {nd }}$ Ed.,

(Chicago: University of Chicago Press) (1983).

Cox, J.P., and Giuli, R.T. Principles of Stellar Structure, Vol. 1, (Gordon and Breach: New York) (1968)

Däppen, W., these proceedings (1994)

Däppen, W., Mihalas, D., Hummer, D.G., Mihalas, B.W. Ap. J., 332, 261 (1988)

Fontaine, G. these proceedings (1994)

Fontaine, G., Graboske, H.C., Jr., and Van Horn, H.M. Ap. J. Supp., 35, 293 (1977)

Graboske, H.C., Jr., Harwood, D. J., and Rogers, F. J. Phys. Rev., 186, 210 (1969)

Hubbard, W.B., and DeWitt, H.E. Ap. J., 290, 388 (1985)

Hummer, D.G., and Mihalas, D. Ap. J., 331, 794 (1988)

Kerley, G.I. Phys. Earth Planet. Inter., 6, 78 (1972)

Lamb, D.Q. PhD Thesis, University of Rochester (1974)

Lamb, D.Q., and Van Horn, H.M. Ap. J., 200, 306 (1975)

Magni, G., and Mazzitelli, I. Astron. Astrophys., 72, 134 (1979)

Marley, M.S., and Hubbard, W.B. Icarus, 73, 536 (1988)

Mazzitelli, I. private communication (1993)

Mihalas, D., Däppen, W., and Hummer, D.G. Ap. J., 331, 815 (1988)

Rogers, F.J., Phys. Rev., A24, 1531 (1981)

Rogers, F.J., these proceedings (1994)

Saumon, D., and Chabrier, G. Phys. Rev. A, 44, 5122 (1991)

Saumon, D., and Chabrier, G. Phys. Rev. A, 46, 2084 (1992)

Saumon, D., Chabrier, G., and Van Horn, H.M. in preparation for Ap. J. (1993)

Saumon, D., and Van Horn, H.M. in Strongly Coupled Plasma Physics, F.J.

Rogers and H. E. DeWitt, Eds. (Plenum: New York), p. 173 (1987)

Stevenson, D.J. Phys. Rev. B, 12, 3999 (1975)

Stevenson, D.J., and Salpeter, E.E. Ap. J. Suppl., 35, 229 (1977) 